\title{
«... Rater encore. Rater mieux. », poétique du ratage chez Jean-François Peyret
}

\section{Victor Thimonier}

\section{(2) OpenEdition}

1 Journals

Édition électronique

URL : https://journals.openedition.org/agon/8983

DOI : $10.4000 /$ agon.8983

ISSN : 1961-8581

Éditeur

Association Agôn

Référence électronique

Victor Thimonier, « « ... Rater encore. Rater mieux. », poétique du ratage chez Jean-François Peyret », Agôn [En ligne], 9 | 2021, mis en ligne le 07 octobre 2021, consulté le 19 janvier 2022. URL : http:// journals.openedition.org/agon/8983; DOI : https://doi.org/10.4000/agon.8983

Ce document a été généré automatiquement le 19 janvier 2022.

Association Agôn et les auteurs des articles 


\title{
"... Rater encore. Rater mieux. ", poétique du ratage chez Jean- François Peyret
}

\author{
Victor Thimonier
}

\begin{abstract}
Comment rater dire comment vu autrement ${ }^{1}$ ?
Samuel Beckett, Cap au pire

Cette propension chez moi à tout laisser filer². Jean-François Peyret, Journal, le « 11 mai 2000 »
\end{abstract}

1 Il y a celui qui rate la marche et celui qui rate sa vie. Pour les deux, il y a des imaginaires théâtraux et des modèles esthétiques. La misère déprimante du second, ses épaules tombantes et ses jambes molles, c'est Beckett. La physionomie désopilante du premier, le regard burlesque qui se retourne sur le pas trébuchant, c'est encore lui. C'est lui qui rate, nous qui regardons et nous n'en ratons rien. "Cap au pire», dit le poète. La littérature de Beckett fait du ratage un modèle, une force majeure communément tragique et joyeuse et donne les clés d'une poétique singulière, inversée, qui transforme l'échec ou l'embuche en moteur de théâtralité.

2 Le metteur en scène et auteur scénique Jean-François Peyret orchestre avec la littérature de Beckett et son imaginaire un dialogue continu mais assourdi, problématique, qui d'années en années, ne semble pas se tarir. Dans le cadre de la compagnie tf2, depuis plus de vingt ans, Jean-François Peyret monte des spectacles qui se confrontent au problème de l'homme, à son tragique et à l'ère scientifique dans laquelle nous vivons. Il le définit comme un théâtre qui « s'expose à la science ${ }^{3}$ ». Il n'y a, sur cette scène, ni vulgarisation, ni tentative de faire des fables à partir de questions scientifiques, mais des stratégies d'invitation pour éprouver, pour construire des expériences de pensée. Ainsi sa scène adresse des invitations à des scientifiques en leur proposant de venir faire de la science d'une autre manière. Sur le plateau du théâtre acteurs, techniques, textes et discours scientifiques se mêlent, se confrontent, se meuvent et tentent ainsi de penser parallèlement. Parmi les références de ces expérimentations, la littérature de Beckett fait toujours figure de pierre d'angle, elle se 
niche dans les plis des discours autant que dans les formes scéniques et participe du geste tragique auquel la scène nous expose. On en retrouve la trace directement dans la composition scénographique et dramaturgique des spectacles mais également avec constance dans le Journal qui accompagne depuis plus de vingt ans le travail de JeanFrançois Peyret et qui est disponible en ligne sur le site de la compagnie tf2 (www.tf2.re). Ce Journal est autant le lieu d'une réflexion dramaturgique sur son processus de création théâtral, qu'un objet littéraire autonome dans lequel le metteur en scène s'interroge sur les problématiques scientifiques contemporaines. Les premiers numéros qui sont consultables datent de 1997-1998, à une époque où le travail de JeanFrançois Peyret s'autonomise de ses collaborations précédentes avec les metteurs en scène Jean Jourdheuil, puis Sophie Loucachevsky, et où son geste scénique s'oriente sensiblement vers la question de la science en scène. C'est alors le temps de nouvelles collaborations qui donnent lieu à plusieurs spectacles où cette question se fait pressante : Un Faust. histoire naturelle, en 1998, avec le biologiste Jean-Didier Vincent ${ }^{4}$; Le Cas Sophie K, en 2005, avec le chercheur en intelligence artificielle Luc Steels ${ }^{5}$ et surtout, la série intitulée: Traité des formes, de 2000 à 2005, avec le neurobiologiste, professeur de la chaire des processus morphogénétiques au Collège de France, Alain Prochiantz

3 Jusqu'en 2007, le Journal n'avait été lu par personne et ce n'est qu'à la faveur des recherches de Julie Valero, et d'une amitié sérieuse qui la lie à Jean-François Peyret, qu'il a commencé à trouver d'abord une puis des lectures. Dans son ouvrage intitulé Le théâtre au jour le jour. Journaux personnels de création de Didier-Georges Gabily, Jean-Luc Lagarce et Jean-François Peyret ${ }^{7}$, Julie Valero, par ailleurs dramaturge au sein de la cie tf2, a longuement commenté l'attitude de diariste du metteur en scène et le rôle de cette production écrite comme œuvre singulière, œuvre hors scène mais dont les pages sont comme « hantées » par la scène ${ }^{8}$.

Dans ce Journal Jean-François Peyret ne cesse de revenir sur ses spectacles comme autant de naufrages spectaculaires. Tragique constat et intime revanche sur les répétitions qui font souffrir, le journal s'écrit, non pas « à sauts et à gambades » comme la figure de Montaigne qui chapeaute son architecture culturelle, mais de ratages en échecs, d'erreurs en vexations. Il nous donne accès à une pensée qui doute, qui se critique, et regarde avec la plus grande distance la scène où elle s'expérimente. Pourtant quelque chose nous dit que dans ces ratages, dans la pensée qui expose ses échecs, tout n'est pas involontaire et que Jean-François Peyret pioche dans les tentatives reprises, ratées, réessayées de Samuel Beckett pour composer la forme de sa scène et éprouver la plasticité de sa pensée. L'écriture, qu'elle soit scénique ou littéraire, tiendrait ainsi le «cap au pire » comme manière d'assurer sa perpétuelle expérimentation.

\section{Beckett en ligne de mire}

4 En 2012, Jean-François Ballay note que Beckett est cité 209 fois dans le Journal ${ }^{9}$ de JeanFrançois Peyret. Il est encore cité à 48 occasions dans les journaux qui couvrent la période 2013-2017 ; avec une insistance particulière en 2013 notamment en lien avec le spectacle Ex vivo / In vitro ${ }^{10}$ et en 2017 autour du spectacle La Fabrique des monstres ou Démesure pour mesure ${ }^{11}$. 
Jean-François Peyret explique cette insistance dans les pages du Journal qui préparent la master-class qu'il consacre au dramaturge irlandais au TNS en 2006 :

Beckett m'a tuer (sic). Je suis assez brouillé avec lui. Au sortir de mon dernier spectacle (Le Cas Sophie K.), mû par quoi, je ne sais, je me suis jeté dans Beckett dont je sentais depuis quelques temps que sa présence (son retour) se faisait pressant dans mon travail. Que des allusions assez plates, il est vrai, à son œuvre palliaient le défaut chez moi de toute nouvelle idée de théâtre. Bref, j'allais buter sur lui. Je décidai alors de le relire (ou de le lire vraiment au fond, - jusque-là j'avais respiré son œuvre parce que son contemporain -, j'avais baigné dans son œuvre, elle me façonnait à mon insu, je ne cherchais pas à savoir comment elle me parlait) [...] Je l'attaquai par l'angle de la technique (à quoi je n'avais pas été consciemment sensible lors de ma première ingestion) $)^{12}$.

6 Au cœur des motifs que la littérature de Beckett amène sur la scène du Journal, le vocabulaire $\mathrm{du}$ ratage fait figure de principale ligne directrice. S'exposent les "défauts ", cette " absence d'idée de théâtre ", cette platitude allusive de la scène, sous le prisme d'un meurtre, d'une brouille irréconciliable et quasiment originaire. Lire ou monter Beckett ne peut être une finalité, il faudrait que ce soit un palliatif. Le seul capable de faire ressentir l'inanité même de l'homme et son dépassement technique. Chez Beckett nul ne sait quoi faire de là d'où il vient, et cette provenance, cette origine d'un corps et d'un être, pèse comme une menace ou une malédiction, entraînant une immanquable vexation qui s'alourdit avec et dans le temps. Chaque vexation provoque des ratages à partir desquels il faut reprendre, réinventer. Beckett agit sur la scène du Journal comme l'un des fondements qui précèdent l'écriture scénique, notamment dans la question des rapports à la technique. Mais le Journal est aussi le temps d'un retour sur l'expérimentation en scène et l'espace où se constatent, se commentent et se prolongent les expériences ratées sur la scène et dans lequel Beckett revient comme interlocuteur privilégié de ces ratages. Ils peuvent être des ratages de laboratoire, des ratages d'apprentissage ou, simplement, des spectacles ratés.

Ainsi, le dernier spectacle du metteur en scène : La Fabrique des monstres ou démesure pour mesure créé en 2018, à partir du roman de Mary Shelley, Frankenstein ou le Prométhée moderne, comme le Journal de 2017 - l'année où ce spectacle prend forme reprennent tant la référence à la littérature du dramaturge irlandais que l'interrogation sur la technique, l'expérimentation et le risque de voir naître une créature ratée.

\section{La créature mal vue mal dite}

7 A priori, rien ne laisse percevoir dans le projet sur le roman gothique de Mary Shelley la présence de Beckett et de son écriture, pourtant toute la scène en dessine les reflets.

8 La scénographie de Nicky Rieti pastiche et détourne notre habitude des imaginaires beckettiens. Sur la scène subsistent les objets typiques de ce «bestiaire» singulier : poubelles, escabeau, trou au centre du plateau. Ces objets répondent parfaitement aux costumes et accessoires tous aussi explicitement beckettiens. L'acteur Jacques Bonnaffé est assis dans une chaise roulante, il porte des lunettes noires, un chapeau melon et une canne d'aveugle; il est le double du Hamm de Fin de partie. Jeanne Balibar fait émerger ses jambes depuis les dessous à travers le trou sur le plateau, puis laisse apparaitre sa tête et son buste. Elle est vêtue de dentelles blanches et rouges et porte une ombrelle et un vaste chapeau. Elle est le double de la Winnie d'Oh les beaux jours. 


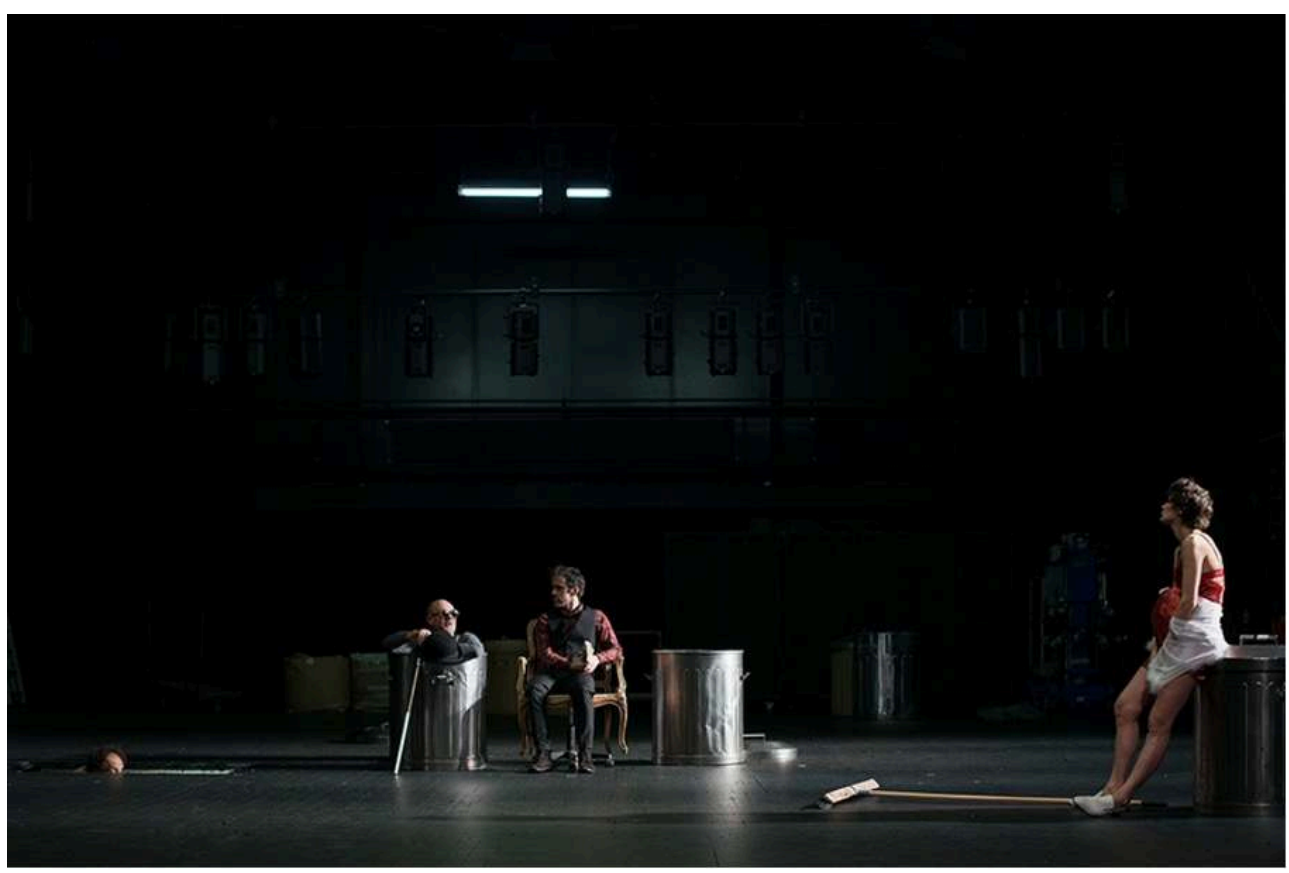

Joël Maillard, Jacques Bonnaffé, Victor Lenoble et Jeanne Balibar - La Fabrique des monstres ou démesure pour mesure, Jean-François Peyret, cie tf2

(c) Mathilde Olmi

Dans le Journal, cette scénographie est qualifiée par le metteur en scène comme «la suite de Fin de partie ${ }^{13}$ » et Peyret avance qu'elle n'est "pas un décor, mais [une] dramaturgie. ${ }^{14}$ » Tout cela produit une «beckettisation de l'ensemble ${ }^{15}$ » qui œuvre d'abord dans le dispositif visuel et qui a également un objectif dramaturgique. Le Journal l'esquisse sous la forme d'une question : « Il y a quelque chose de pourri dans les

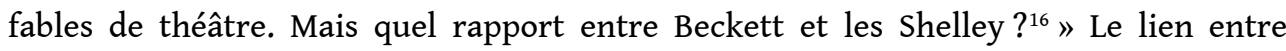
l'objet du spectacle et la référence à Beckett est directement issu de la problématique du ratage et de ce qui est, ou peut devenir, «pourri ».

Dans le roman de Mary Shelley, le docteur Victor Frankenstein compose une créature. Il donne naissance à un être qui tout entier lui échappe. Née de fragments de cadavres, de morceaux rapiécés d'êtres, la créature est hideuse et sa naissance, tout aussi effrayante qu'hallucinante. Là où la science devrait produire un être parfait, elle produit une créature ratée et le long processus expérimental du docteur, que le roman retrace abondamment, n'y change strictement rien.

En préparant le spectacle, Peyret réfléchit au personnage du docteur et note dans son journal : « Il [Victor] veut connaitre le secret du vivant, et en bon baconien, connaître c'est expérimenter. Connaitre le secret de la vie, c'est pouvoir la fabriquer. On pourrait même se dire que reproduire la vie, la créer est plus important que la comprendre. Contemplation et hybris (sic.) ${ }^{17}$. $»$ Le docteur est animé par cette fabrication, il est ivre de son pouvoir, mais cette fabrication ne se réalise qu'au prix de l'horreur. La créature qu'il finit par faire, issue d'une expérience de laboratoire, avide de reconnaissance et d'amour va, à mesure que la fable se déploie, se révéler un fléau pour son créateur. Elle assassine ses proches, dérange le cours de son existence, résiste et harcelle celui qui l'a conçue. Toutefois, comme le laisse voir Mary Shelley dans le roman - et Peyret y insiste 
également-, elle n'est pas viscéralement mauvaise et fait plutôt l'apprentissage du tragique et de la violence. Dans son Journal le metteur en scène écrit : «La créature est sans nom, c'est peut-être cela sa monstruosité. Imaginez un peu. Que du coup une des questions essentielles, c'est l'apprentissage. Et la fabrique de l'assassin. ${ }^{18}$ » Ainsi, la créature est rendue mauvaise par l'abandon inaugural qu'elle subit, par la vexation considérable de sa non appartenance à l'humanité dont elle fait progressivement et intensément le constat: "J'étais seul. Je me souvenais de la supplication lancée par Adam à son Créateur. Mais où était donc le mien? Il m'avait abandonné et, l'amertume au cœur, je le maudissais. ${ }^{19}$ "

Joël Maillard aux prises avec la créature

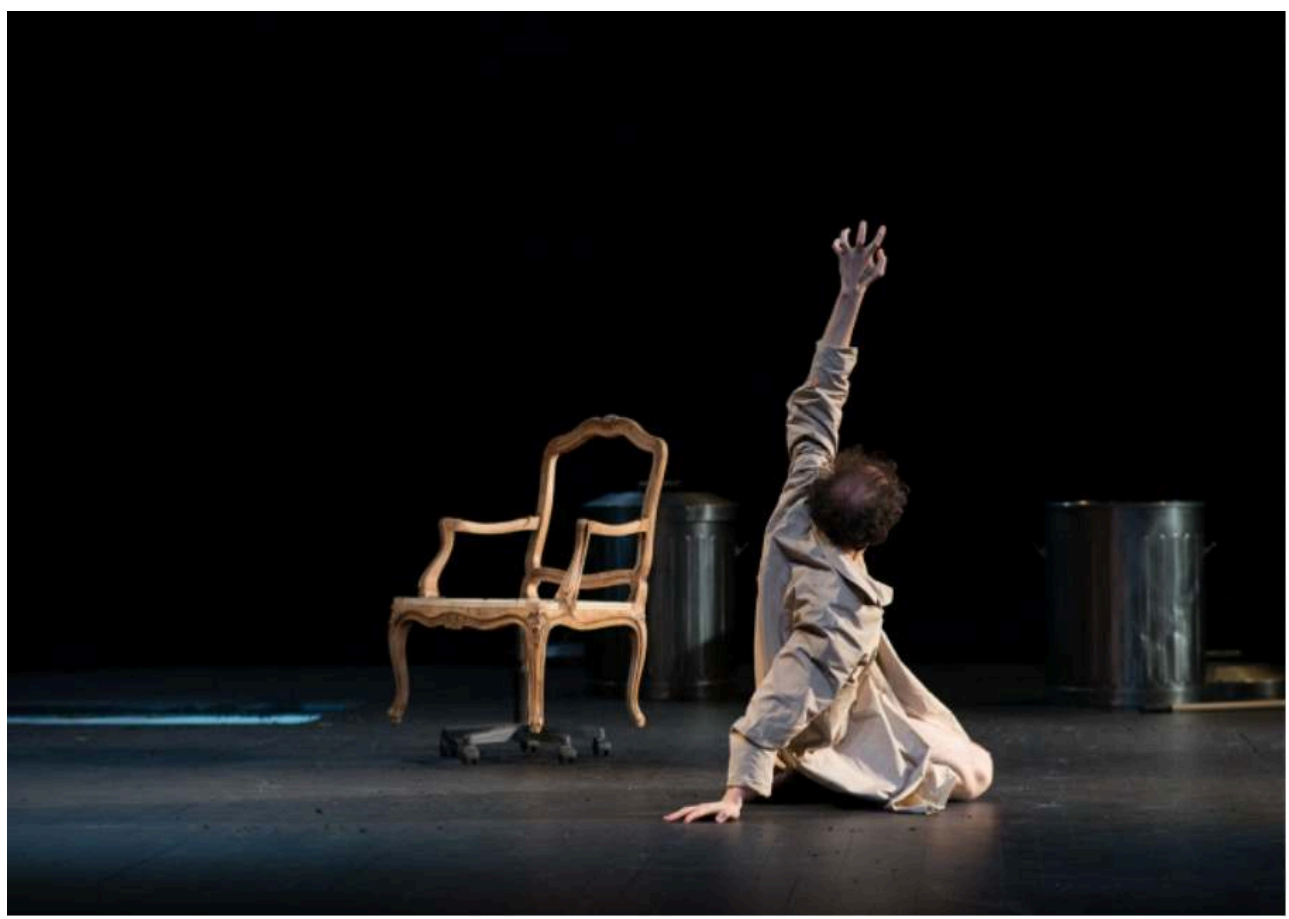

Joël Maillard - La Fabrique des monstres ou démesure pour mesure, Jean-François Peyret, cie tf2 (c) Mathilde Olmi

Née sans fécondation, née en laboratoire, née sans mère, née de l'expérimentation, la créature n'appartient pas aux hommes. Cette vexation, qui est aussi une exclusion, se répète d'ailleurs dans le roman lorsque la créature est abandonnée et haïe, une seconde fois, par la famille où elle avait cru bon de trouver refuge et auprès de laquelle elle a été amenée sur les chemins de la parole et de la pensée.

13 La situation de cette créature ratée est donc dans un temps mort, un temps où la difformité, le handicap originaire et génital empêche d'envisager le commencement de quoi que soit d'autre qu'un apprentissage de la destruction et de la violence. Dans ce temps mort - ou ce temps propice à la mise à mort - où elle évolue, la créature sans nom issue du laboratoire de Victor Frankenstein retrouve Hamm de Fin de Partie. Son discours double d'ailleurs directement le personnage beckettien, il maudit de la même manière son origine :

Jour détestable que celui où je reçus la vie! m'écriai-je au comble du tourment. Maudit créateur! Pourquoi avoir formé un monstre à ce point hideux que même toi, de dégoût, tu te détournas de moi ? Dieu, en sa miséricorde, rendit l'homme beau et 
attirant, à son image; mais mon corps n'est que l'image infecte du tien, et notre ressemblance même le rend plus horrible encore. Satan avait ses compagnons, des démons comme lui, qui l'admiraient et l'encourageaient. Moi, je suis solitaire et exécré. ${ }^{20}$

$14 \mathrm{Au}$ «Maudit progéniteur ${ }^{21}$ !» de Hamm dans Fin de partie, répond le «Maudit créateur!» de la créature de Frankenstein. Comme son ancêtre de laboratoire, Hamm est handicapé. Si le premier court et saute des montagnes, étant par cela proprement inhumain, l'autre ne bouge plus. Il est amoindri, figé dans son fauteuil entre les temps, coincé dans les limbes du même continûment répété, à la limite de sa propre disparition. Les deux personnages sont frappés par la vexation d'être; ratés en tout point. Tous deux font face à ce "vrai regard méchant sur la vie: je veux dire impitoyable ${ }^{22}$ » par lequel, dix ans plus tôt, Jean-François Peyret résume la dramaturgie de Beckett dans les pages de son Journal de 2007.

Dans La Fabrique des monstres, Peyret exploite cette situation semblable, il en provoque l'écho continu, par la scénographie et le corps des acteurs d'abord, mais également par le tempo et le rythme des séquences qui alanguissent la scène, tout en y provoquant des sursauts de rire grinçant. Peu à peu, c'est le spectacle qui paraît devenir la créature et la «beckettisation de l'ensemble » transforme la scène du roman en scène suspendue entre les temps. Tout glisse dans ce hors temps de la dramaturgie beckettienne où le rire et l'ennui se confondent et s'informent, se regardent et se jaugent. Pour le spectateur, l'expérience est étrange et produit une attention syncopée. Comme chez Beckett, le rire ne surgit pas franchement mais s'inscrit dans la lenteur de la répétition. Il est le produit d'une ironie sur la possibilité de tenir un discours, de croire aux histoires ou aux productions de la science. Il est un rire tragique, une «tragédie rigolarde ${ }^{23}$ » qui ne commence plus rien que sa propre itération. Comme la naissance problématique de la créature, comme la naissance catastrophique de Hamm, la scène est soumise à la catastrophe de sa présence, maintenant qu'elle est là, il faut bien l'occuper, en occuper son temps. Ainsi Peyret retourne le problème de la fable du roman en un problème de composition du spectacle, en un principe poétique.

Pour que la créature de Victor Frankenstein existe sur la scène, il faut qu'elle échappe. Pour que le spectacle naisse, il faut ne plus être en mesure de le contrôler. Enfin, pour que le monstre ait un autre visage que celui de Boris Karloff qui colonise les imaginaires de l'œuvre, il faut en rater la représentation. La créature ne doit exister que sous une forme ratée, qu'en tant qu'expérience continuelle et souvent décevante. Le ratage de sa représentation devient une des actions fondamentales du spectacle. Il faut que le spectacle s'autonomise, déçoive, hante, effraie, tue son spectateur, pour être à la mesure de la créature du roman. Cela passe alors par la machine sonore proposée sur scène et qui, encore une fois, fait écho au devenir machinique de l'homme sur la scène beckettienne.

17 Avec le compositeur Daniele Ghisi et en lien avec l'IRCAM, le spectacle propose de substituer au monstre de Mary Shelley et à sa silhouette cinématographique convenue, un monstre sonore qui déborde et se libère du cadre dans lequel il est invité à naître.

...l'idée est venue de mettre en place une machine musicale, la Machine (comme on dit la Créature) qui apprenne à faire elle-même de la musique à partir, certes, des instructions qu'elle reçoit mais à mesure que cet apprentissage "tourne“, la maîtrise du résultat, l'écriture si on veut, échappe imprédictiblement au compositeur [...] 
Ce travail s'inscrit dans le contexte actuel de la recherche en informatique dominée par les Big Data et l'apprentissage profond (deep learning) et pourrait constituer une première réplique artistique à la toute-puissance algorithmique, au risque de s'y prendre. Victor Frankenstein sait de quoi nous parlons. Et il n'y a pas d'art sans risque, comme on $\operatorname{dit} . .^{24}$

Le temps réel et l'apprentissage de la machine se rencontrent et déterminent la temporalité propre du spectacle en même temps que sa dramaturgie. C'est la naissance et l'apprentissage d'une machine qui constituent l'écriture monstrueuse proposée aux acteurs et aux spectateurs. La machine écrit en direct et n'est pas contrôlable par l'équipe du spectacle : pleinement expérimentale, elle s'autorise à rater. Julie Valero le note d'ailleurs lorsqu'elle témoigne des perspectives ouvertes par cette machine :

Ce résultat, justement, on ne peut le connaître d'avance, et il sera différent à chaque représentation. Le champ des variations possibles est très grand : on peut se dire que l'on peut obtenir un discours musical recevable, acceptable, consommable pour l'auditeur, mais que ça peut se gâter jusqu'à devenir insupportable, terrible, monstrueux (ou sublime? La question du sublime étant tapie derrière toute cette histoire) $)^{25}$.

Dans le spectacle cette «Machine » qui échappe, comme la créature ratée de Victor Frankenstein, rate également sa représentation. Contrairement à la créature capable d'apprendre à parler, à aimer, à haïr, la machine développée par Daniele Ghisi ne parvient pas à étonner la représentation dans laquelle elle intervient. Jean-François Peyret le supposait déjà dans son Journal au moment des premières répétitions du projet:

Hier à l'IRCAM, studio 6, pour être précis. Inquiétude au début : ce que Daniele (Ghisi) et Robin (Meier) nous font écouter ne semble pas très exploitable (spectaculaire), surtout s'agissant des paroles. L'effet n'est guère plus intéressant que du grommelo. Le spectateur de théâtre n'y trouvera pas son compte, et ne verra même pas la malice technologique, ou la prouesse. Va lui faire comprendre que c'est compliqué à fabriquer ${ }^{26 !}$

Sans contrôle sur les fruits de son apprentissage, elle ne produit pas suffisamment de matière pour venir nourrir le spectacle, pour être «spectaculaire». Bien que Daniele Ghisi l'ait abreuvée de tout Schubert, de Beethoven, de Brahms, etc... que la Machine apprend, elle ne parvient pas à produire sa propre singularité sonore et n'intervient, en définitive, qu'assez peu dans le projet final. C'est donc, par une étrange inversion involontaire, que le spectacle finit par parvenir à son objectif en créant, dans et par l'échec, la réplique sans doute la plus sérieuse des expérimentations du docteur. C'est bien parce que le spectacle rate sa représentation de la créature, qu'il reproduit le même geste que celui du docteur et que celui de Beckett. Comme Victor Frankenstein, l'objectif technoscientifique, transposé ici en objectif techno-scénique, crée un monstre; l'envers de ce qui était prévu. Comme chez Beckett, la fable scénique n'est plus que la répétition de son ratage. Dans son Journal, Jean-François Peyret referme ses réflexions sur $L a$ Fabrique des monstres avec ce constat lucide et tragique sur le spectacle : «On voit déjà à quoi il ressemble, ou plutôt à quoi il ne ressemble pas. Un de ses atouts, cela a déjà été remarqué, c'est qu'il ne ressemble à rien, qu'il reste non identifiable ou non identifié. ${ }^{27}$ "

21 Ainsi, dans la variation sur le Frankenstein apparaît et se concrétise le risque de l'expérimentation scénique mais le Journal évite de copier l'attitude de rejet tenue par le docteur dans le roman. Sans hybris, Jean-François Peyret reconnaît et accepte la créature qui se propose à lui : "Donc il est un peu comme ça et nous ne pouvons 
complètement renier notre progéniture, même s'il est possible de l'avoir rêvée autre... ${ }^{28}$ »

\section{Démesure pour mesure}

Démesure pour mesure est le sous-titre du spectacle sur Frankenstein et c'est dans l'architecture de la démesure que le projet se compose, une démesure qui confine à l'échec de toute l'entreprise et à l'angoisse de celui qui la porte. Une démesure expérimentale que le Journal autant que la scène savent donner à lire à ceux qui s'y attardent.

Peut-être que l'usage fait de Beckett, de son bestiaire et du rythme si particulier de sa scène, permet de faire émerger "une jubilation jointe à la connaissance de la catastrophe ${ }^{29}$ » comme le dit Clément Rosset. Peut-être également que cette jubilation, chez Jean-François Peyret, provient de l'expérience du ratage, que c'est face à lui que l'invention se meut avec plus de force, que la scène peut gagner sa puissance d'expérience scientifique. Alain Prochiantz commente d'ailleurs cette démarche scientifique et expérimentale dans le texte paru à la suite du spectacle La Génisse et le Pythagoricien qu'il a réalisé avec Jean-François Peyret :

En clair, le théâtre n'était qu'un prétexte pour poursuivre dans mon vice du "pas de côté", ne pas être dedans sans être dehors. Sur le bord, ce bord intérieur, fissure où tout s'invente, tout se joue. Ratage et banco!

Je n'irai pas plus loin, mais je crois que le rapport de Peyret au théâtre relève de la même perversité. Même dans le ratage, l'inventivité se perçoit. Expérience, expérience, expérience! Après Jean-François, il est difficile de ne pas trouver le théâtre, l'autre théâtre, ennuyeux. Il n'y a pas de pensée sans risque. ${ }^{30}$

Il est peut-être abusif d'imputer à l'écriture scénique de Jean-François Peyret quelque chose comme une poétique du ratage qui guiderait, en familier de la littérature de Beckett, tous ses objectifs de création. Il faut le reconnaître, rater c'est aussi, parfois, seulement rater et pas toujours, dans le méta du méta, parvenir à la clarté la plus singulière. Toutefois, il est certain qu'il y a, dans la quête ontologique de ce théâtre une tentative d'expérimenter, de négocier voire de réagir au ratage de l'homme et à son perpétuel tragique. Rater s'inscrit donc ici dans le processus d'écriture et le ratage à l'œuvre dans le texte de Mary Shelley - comme dans la référence à Beckett - nourrit toutes les actions scéniques. En même temps, c'est ce ratage qui informe le tragique propre à ce théâtre. Le jeu entretenu avec les différentes possibilités de rater ou la reconnaissance d'un objet, d'une scène, d'un spectacle raté constitue ainsi le jeu de l'écriture scénique si particulière du metteur en scène. Jean-François Peyret résume d'ailleurs la trajectoire évolutive de l'être humain que ses spectacles traitent en des termes on ne peut plus clairs au cœur du livre La Génisse et le Pythagoricien : «L'homme est un animal raté, et c'est cet échec qui a fait sa réussite. ${ }^{31}$ » Dans l'expérience de l'écriture, sur la scène ou dans le Journal, c'est le même jeu d'échec et de ratage, jeu expérimental, qui propose un peu d'humanité à cette créature étrange qu'est le théâtre. 


\section{BIBLIOGRAPHIE}

BALLAY, Jean-François, Disparition de l'homme et machinerie humaine sur la scène contemporaine Denis Marleau, Heiner Goebbels, Jean-François Peyret, thèse de doctorat, sous la direction de MarieMadeleine Mervant-Roux, soutenue publiquement le 12 novembre 2012, à l'Université Paris 3 Sorbonne Nouvelle.

BECKETT, Samuel, Fin de partie, Paris, éd. de Minuit, 1975, 128 p.

BECKETT, Samuel, Cap au pire, trad. Edith Fournier, Paris, éd. de Minuit, 1991, 61 p.

PEYRET, Jean-François, Journal 1998-2017. Disponible en ligne sur le site de la compagnie tf2. [URL : http://www.tf2.re]

PEYRET, Jean-François et PROCHIANTZ, Alain, La Génisse et le Pythagoricien - Traité des formes I, Paris, éd. Odile Jacob, 2002, 254 p.

PEYRET, Jean-François, « Le théâtre et la recherche scientifique. », entretien avec Franck Renucci, revue Hermès, 2015/2, n 72, p. 140.

ROSSET, Clément, Le Réel. Traité de l'idiotie, Paris, éd. de Minuit, 2011, 155 p.

SHELLEY, Mary, Frankenstein ou le Prométhée moderne, trad. Alain Morvan, Gallimard, Folio, 2015, $336 \mathrm{p}$.

VALERO, Julie, Le théâtre au jour le jour. Journaux personnels de création de Didier-Georges Gabily, JeanLuc Lagarce et Jean-François Peyret, Paris, L'Harmattan, 2013, 244 p.

\section{NOTES}

1. Samuel Beckett, Cap au pire, trad. Edith Fournier, Paris, éd. de Minuit, 1991, p. 4

2. Jean-François Peyret, Journal 1998-2000, « le 11 mai 2000 », p. 158. Disponible en ligne sur le site de la compagnie tf2, consulté le 10/06/2020. [URL : http://www.tf2.re/ Peyret_journal/Peyret_Journal1998_2000.pdf]

3. « Il m'est donc apparu impossible d'esquiver les questions que posent à notre temps la ou les sciences ou les technosciences, et impossible de ne pas exposer mon théâtre (" exposer », comme on s'expose à un risque ou au soleil) à ces questions qui configurent le tragique de notre temps, et son caractère indépassable. ", in JeanFrançois Peyret, «Le théâtre et la recherche scientifique. », Entretien avec Franck Renucci, Revue Hermès, 2015/2 ( ${ }^{\circ}$ 72), p. 140.

4. Jean-François Peyret et Jean Didier Vincent, Un Faust. histoire naturelle, d'après Goethe, créé à la MC93 en 1998.

5. Jean-François Peyret, Le Cas Sophie K, sur la mathématicienne Sophie Kovalevskaïa, avec Luc Steels, création Festival d'Avignon, 2005.

6. Jean-François Peyret et Alain Prochiantz, le cycle du Traité des formes est un triptyque composé de 2000 à 2005. Il comprend : La Génisse et le Pythagoricien (Traité des formes I), d'après Ovide, créé au Théâtre national de Strasbourg en 2002 ; Des chimères en automne (Traité des formes II), sur Darwin, créé au Théâtre national de Chaillot en 2003 et Les Variations Darwin (Traité des formes III), créé au Théâtre national de Chaillot en 2005. 
7. Julie Valero, Le théâtre au jour le jour. Journaux personnels de création de Didier-Georges Gabily, Jean-Luc Lagarce et Jean-François Peyret, Paris, L'Harmattan, 2013, 244 p.

8. Voir notamment « 3. Jean-François Peyret : La question de l'œuvre. », in Julie Valero, Le théâtre au jour le jour., op. cit., p. 191 à 228.

9. Jean-François Ballay, Disparition de l'homme et machinerie humaine sur la scène contemporaine - Denis Marleau, Heiner Goebbels, Jean-François Peyret, thèse de doctorat, sous la direction de Marie-Madeleine Mervant-Roux, soutenue publiquement le 12 novembre 2012, p. 210.

10. Jean-François Peyret et Alain Prochiantz, Ex Vivo / In vitro, mise en scène JeanFrançois Peyret, La Colline - Théâtre National, Paris, 2011.

11. Jean-François Peyret, La Fabrique des montres ou Démesure pour mesure, création Théâtre de Vidy Lausanne, janv. 2018.

12. Jean-François Peyret, Journal 2006, « 2 août 2006 », p. 88.

13. Jean-François Peyret, Journal 2017, « le 13 septembre », p. 63.

14. Ibidem.

15. Jean-François Peyret, Journal 2017, « le 14 mars », p. 22.

16. -, Journal 2017, « le 13 septembre », p. 63.

17. -, Journal 2016, « le 3 août », p. 69.

18. -, Journal 2017, « le 21 janvier $2018 »$, p. 117.

19. Mary Shelley, Frankenstein ou le Prométhée moderne, trad. Alain Morvan, Gallimard, Folio, 2015, [ePub - format numérique], p. 324.

20. Mary Shelley, Frankenstein ou le Prométhée moderne, op. cit., p. 321.

21. Samuel Beckett, Fin de partie, Paris, éd. de Minuit, 1975, p. 23.

22. Jean-François Peyret, Journal 2007, « 13 janvier », p. 7.

23. "Cette vie est sans pitié ; ma lecture de Beckett, une tragédie rigolarde. », JeanFrançois Peyret, Journal 2007, « le 13 janvier », p. 7.

24. Jean-François Peyret, in « En collaboration avec l'IRCAM », La Fabrique des monstres ou démesure pour mesure, livre interactif d'accompagnement du spectacle, édité par le Théâtre de Vidy Lausanne, textes et choix des extraits Julie Valero, disponible en ligne, consulté le 11/07/2020, [URL : https://vidy.ch/sites/default/files/

notes_autour_de_la_fabriques_des_monstres-ev.pdf].

25. Ibidem.

26. Jean-François Peyret, Journal 2017, « le 14 mars », p. 22.

27. Ibidem., « le 29 janvier 2018 », p. 120.

28. Ibidem., p. 121.

29. Clément Rosset, Le Réel. Traité de l'idiotie, Paris, éd. de Minuit, 2011, p. 75.

30. Alain Prochiantz, in Jean-François Peyret et Alain Prochiantz, La Génisse et le Pythagoricien - Traité des formes I, Paris, éd. Odile Jacob, 2002, p. 49.

31. Ibidem., p. 115. 


\section{RÉSUMÉS}

Dans le cadre de la création du spectacle La Fabrique des monstres ou Démesure pour mesure (2017) le metteur en scène Jean-François Peyret construit un parallélisme entre l'œuvre de Samuel Beckett et celle de Mary Shelley avec son Frankenstein. Dans ce travail, rater la créature monstrueuse devient un enjeu essentiel de la pratique scénique et ce ratage laisse des traces sur la scène et dans les journaux du metteur en scène.

\section{INDEX}

Mots-clés : Rater, Frankenstein, processus de création, écriture scénique, Beckett (Samuel), Peyret (Jean-François), Shelley (Marie)

\section{AUTEUR}

\section{VICTOR THIMONIER}

Victor Thimonier est ATER à l'Université Evry Val d'Essonne, docteur en études théâtrales de l'Université de Paris Nanterre, ancien élève de l'ENS de Lyon et du Master Pro Mise en scène et dramaturgie - Paris Nanterre. 